\title{
Influence of glycemic control on some real-time biomarkers of free radical formation in type 2 diabetic patients: An EPR study
}

\author{
Veselina Georgieva Gadjeva ${ }^{1}$, A, D, F, Petia Goycheva ${ }^{2, A-E}$, Galina Nikolova ${ }^{3, B-D}$, Antoaneta Zheleva ${ }^{3, D, E}$ \\ ${ }^{1}$ Medical Faculty, Trakia University, Stara Zagora, Bulgaria \\ ${ }^{2}$ Department of Propedeutics of Internal Diseases, Medical Faculty, Trakia University Hospital, Stara Zagora, Bulgaria \\ ${ }^{3}$ Department of Chemistry and Biochemistry, Medical Faculty, Trakia University, Stara Zagora, Bulgaria \\ A - research concept and design; $\mathrm{B}$ - collection and/or assembly of data; $\mathrm{C}$ - data analysis and interpretation; \\ $\mathrm{D}$ - writing the article; $\mathrm{E}$ - critical revision of the article; $\mathrm{F}$ - final approval of the article
}

\section{Address for correspondence}

Veselina Georgieva Gadjeva

E-mail: vgadjeva@mf.uni-sz.bg

\section{Funding sources}

None declared

\section{Conflict of interest}

None declared

Received on December 8, 2015

Reviewed on April 28, 2016

Accepted on February 14, 2017

\section{Abstract}

Background. The pathology of diabetes is associated with several mechanisms, one of which is oxidative stress (OS). The relationship between OS and diabetic complications has been extensively investigated. OS has been suggested to be involved in the genesis of both macro- and microangiopathy. In contrast, the relationship between $0 S$ and insulin action is a neglected research area.

Objectives. The aim of this study is to elucidate the effect of glycemic control in type 2 diabetic patients by following the serum levels of some real-time oxidative stress biomarkers.

Material and methods. The study group consisted of 53 type 2 diabetic patients (31 with poor glycemic control and 22 with good glycemic control) and 24 healthy control subjects. The oxidative stress biomarkers $($ ROS, Asc and $\bullet N O)$ were measured by using electron paramagnetic resonance spectroscopy (EPR) methods and compared with clinical parameters.

Results. The statistically significantly higher levels of ROS products and $\bullet N O$ in type 2 diabetic patients in both groups compared to controls mean that the oxidation processes take place at the time the survey is performed. Free radical overproduction persists after the normalization of the glucose levels, and oxidative stress may be involved in the "metabolic memory" effect. This is confirmed by the positive correlation between ROS levels/.NO and average blood glucose levels, triglycerides, and total cholesterol. Furthermore, the low level of the ascorbate radical in both diabetes groups compared to controls confirmed an increase in oxidation processes.

Conclusions. Higher levels of real-time biomarkers show that intensive insulin treatment does not lead to the expected decrease in oxidative processes involving ROS and •NO, probably due to "metabolic memory".

Key words: diabetes, oxidative stress, free radicals

DOI

10.17219/acem/68988

\section{Copyright}

Copyright by Author(s)

This is an article distributed under the terms of the

Creative Commons Attribution Non-Commercial License

(http://creativecommons.org/licenses/by-nc-nd/4.0/) 
Under normal conditions, the low concentrations of reactive oxygen species (ROS) and reactive nitrogen species (RNS) are essential for life, because they are involved in cell signaling, and are used by phagocytes for their bactericidal action. Cells are protected against the toxic effects of high concentrations of ROS by a balanced level of endogenous enzymatic and non-enzymatic antioxidants. Oxidative stress (or oxidant-derived tissue injury) occurs when the production of oxidants or reactive oxygen species (ROS) exceeds local antioxidant capacity. When this occurs, the oxidation of important macromolecules including proteins, lipids, carbohydrates, and DNA ensues. ${ }^{1}$

It has been suggested that oxidative stress (OS) can act as a contributing factor in the pathogenesis of many diseases. ${ }^{2}$ The pathology of diabetes is not well established, and it is associated with several mechanisms, one of which is OS. OS plays an important role in the pathogenesis of diabetes, and leads to a number of complications. Therefore, several studies suggest that diabetic patients are chronically exposed to the influence of oxidative stress. ${ }^{3,4}$

ROS generation and free radical formation are increased in patients with diabetes mellitus. ${ }^{5,6}$ Free radicals generated through glycation may accelerate the oxidation reactions in the vascular walls, thereby contributing significantly to the pathogenesis of diabetic angiopathy. ${ }^{7-10}$ Hyperglycemia-induced overproduction of superoxide is the causal link between high glucose and the pathways responsible for hyperglycemic damage. Through the use of complex laboratory equipment, it has been shown that the samples taken from patients with type 2 diabetes have increased levels of lipids, DNA, DNA bases and proteins. Compared to healthy controls, the values of these indicators are much over the normal values and indicate the presence of OS in those patients. However, because free radicals are highly unstable and reactive, and difficult to measure in vivo, most experiments are performed in vitro or ex vivo. Free radicals have unpaired electrons, which can be detected selectively and sensitively by electron spin resonance (ESR) spectroscopy. ${ }^{11}$ In the current study, to the best of our knowledge, we explored for the first time the influence of the glycemic control on some real-time biomarkers of free radical formation in type 2 diabetic patients, using the EPR spectroscopy technique. For this purpose, we investigated the levels of ROS, Asc • and •NO radicals in the serum of 2 experimental groups, with poor glycemic control before starting active insulin therapy and with good glycemic control, and in healthy controls.

\section{Material and methods}

This study included 53 samples from patients suffering from type 2 diabetes mellitus (age-matched male/female subjects) divided into 2 groups: with poor glycemic control $(\mathrm{n}=31)$ and with good glycemic control $(\mathrm{n}=22)$ and healthy volunteers as controls $(\mathrm{n}=24)$ (Table 1$)$. The study was carried out at the Clinic of Endocrinology, Prof. Stoyan Kirkovich University Hospital, Stara Zagora, Bulgaria. The general characteristics of age, sex, alcohol, diet and smoking, particularly related to preferences, were investigated by a selfadministered questionnaire. As a criterion for good or poor glycemic control, we used the guidance of EASD: fasting plasma glucose (FPG) 4.0-6.1 mmol/L and HbA1c $\leq 7 \%$.

In the diabetic group with good glycemic control, 14 patients were treated with oral hypoglycemic agents (sulphonylureas and biguanides), 4 were insulin-treated, and 4 with a combination of insulin plus biguanides. In the group of diabetic patients with poor glycemic control, 9 were treated with oral hypoglycemic agents (sulphonylureas plus biguanides), 9 were insulin-treated, 13 with the combination

Table 1. Details of the patients

\begin{tabular}{|c|c|c|c|}
\hline Variables & $\begin{array}{l}\text { Control } \\
n=24\end{array}$ & $\begin{array}{l}\text { Patients with good } \\
\text { glycemic control } \\
\qquad n=22\end{array}$ & $\begin{array}{l}\text { Patients with poor glycemic control } \\
\qquad n=31\end{array}$ \\
\hline Age & $48.44 \pm 8.74$ & $60.91 \pm 10.88$ & $61.50 \pm 11.29$ \\
\hline $\operatorname{Sex}(m / f)$ & $13 \mathrm{M} / 11 \mathrm{~F}$ & $10 \mathrm{M} / 12 \mathrm{~F}$ & $20 \mathrm{M} / 11 \mathrm{~F}$ \\
\hline $\mathrm{HbA1c}(\%)$ & $5.60 \pm 0.85$ & $\begin{array}{l}\quad 6.30 \pm 0.71 \\
p<0.00 \text { vs control }\end{array}$ & $\begin{array}{c}9.70 \pm 1.50 \\
p<0.00 \text { vs control } \\
p<0.00 \text { vs good glycemic control }\end{array}$ \\
\hline FBG $(\mathrm{mmol} / \mathrm{L})$ & $5.27 \pm 0.57$ & $5.43 \pm 0.89$ & $\begin{array}{c}12.72 \pm 5.15 \\
p<0.00 \text { vs control } \\
p<0.00 \text { vs good glycemic control }\end{array}$ \\
\hline Cholesterol (mmol/L) & $4.04 \pm 0.2$ & $\begin{array}{c}4.88 \pm 0.2 \\
p<0.00 \text { vs control }\end{array}$ & $\begin{array}{c}5.35 \pm 0.2 \\
p<0.00 \text { vs control }\end{array}$ \\
\hline Triglycerides (mmol/L) & $1.31 \pm 0.15$ & $\begin{array}{c}2.13 \pm 0.2 \\
p<0.00 \text { vs control }\end{array}$ & $\begin{array}{c}2.65 \pm 0.2 \\
p<0.00 \text { vs control }\end{array}$ \\
\hline HDL cholesterol (mmol/L) & $0.91 \pm 0.019$ & $1.19 \pm 0.04$ & $\begin{array}{c}1.32 \pm 0.05 \\
p<0.00 \text { vs control }\end{array}$ \\
\hline LDL cholesterol (mmol/L) & $2.03 \pm 0.11$ & $\begin{aligned} & 2.72 \pm 0.24 \\
p< & 0.00 \text { vs control }\end{aligned}$ & $\begin{array}{c}2.96 \pm 0.2 \\
p<0.00 \text { vs control }\end{array}$ \\
\hline
\end{tabular}


of drugs (insulin plus biguanides). Members of the control group were selected from people without family history of either diabetes or dyslipidemia, and with normal thyroid, hepatic and renal functions. The venous blood of the diabetic patients and controls was collected in the morning after an overnight fast. Informed consent was obtained from all participants in the study, according to the ethical guidelines of the Helsinki Declaration (1964). The clinical data of the patients examined in this study are presented in Table 1.

\section{Electron paramagnetic resonance (EPR)}

All EPR measurements were carried out on a Bruker spectrometer equipped with a standard resonator. The results are expressed in arbitrary units and performed by using Simfonia software and Bruker WIN-EPR. All chemicals and spin-traps, i.e., N-tert-butyl-alpha-phenylnitrone (PBN) and 2-(4-carboxyphenyl)-4,4,5,5-tetra-methylimidazoline-1oxyl-3-oxide (Carboxy-PTIO.K), were purchased from Sigma-Aldrich, Germany and were analytical grade.

\section{EPR ex vivo evaluation of ascorbate radicals}

The Asc $\bullet$ measurement was according to Bailey with some modification. ${ }^{12}$ Briefly, the serum from the patients and volunteers were mixed with DMSO at 1:3 ratios, and centrifuged for $10 \mathrm{~min}$ at $4000 \mathrm{rpm}$. Then, the supernatant was immediately transferred and measured. The EPR settings were $3505.00 \mathrm{G}$ center field, $20.00 \mathrm{~mW}$ microwave power, $1.00 \mathrm{G}$ modulation amplitude, $15 \mathrm{G}$ sweep width, $1 \times 10^{5}$ gain, $40.96 \mathrm{~ms}$ time constant, $60.42 \mathrm{~s}$ sweep time, 10 scans per sample.

\section{Ex vivo ROS evaluation}

The ROS level measurement was according to Shi et al. with some modification. ${ }^{13}$ The real-time formation of ROS in the serum was investigated by mixing the samples with PBN spin trapping. The EPR settings were $3503.73 \mathrm{G}$ center field, $20.00 \mathrm{~mW}$ microwave power, $5 \mathrm{G}$ modulation amplitude, $50 \mathrm{G}$ sweep width, $1 \times 10^{5}$ gain, $81.92 \mathrm{~ms}$ time constant, 125.95 s sweep time, 5 scans per sample.

\section{EPR ex vivo evaluation of $\bullet N O$ radicals}

Based on the methods published by Yoshioka et al. and Yokoyama et al., we developed and adapted the EPR method for evaluating the levels of $\bullet \mathrm{NO}$ radical in the serum. ${ }^{14,15}$ Briefly, a $50 \mu \mathrm{M}$ Carboxy-PTIO.K solution was dissolved in a mixture of $50 \mathrm{mM}$ Tris (pH 7.5) and DMSO at a ratio 9:1. To $100 \mu \mathrm{L}$ of serum, $900 \mu \mathrm{L}$ of tris buffer dissolved in DMSO (9:1) was added and centrifuged for $10 \mathrm{~min}$ at $4000 \mathrm{rpm}$ at $4^{\circ} \mathrm{C}$. To record the spin adduct between Carboxy-PTIO and generated $\bullet \mathrm{NO}$, we mixed a $100 \mu \mathrm{L}$ sample and $100 \mu \mathrm{L}$ $50 \mathrm{mM}$ solution Carboxy-PTIO. The EPR settings were $3505 \mathrm{G}$ centerfield, $6.42 \mathrm{~mW}$ microwave power, $5 \mathrm{G}$ modu- lation amplitude, $75 \mathrm{G}$ sweep width, $2.5 \times 10^{2}$ gain, $40.96 \mathrm{~ms}$ time constant, $60.42 \mathrm{~s}$ sweep time, 1 scan per sample.

\section{Statistical analysis}

The results are expressed as mean \pm SE. Statistical analysis was performed with STATISTICA v. 7 software (StatSoft, Inc., Tulsa, USA). Statistical analysis was performed using Student's t-test, independent, by groups. A p-value $<0.05$ was considered as significant.

\section{Results}

The results obtained for the ROS levels measured in all groups (Fig. 1), in the serum of patients with poor glycemic control before intensive insulin applications were statistically significantly higher than in the controls (mean $2.15 \pm 0.07$ vs mean $0.68 \pm 0.06$, p $<0.0001$, t-test). In patients with good glycemic control, the ROS levels were also statistically significantly increased compared to the controls (mean $2.20 \pm 0.08, \mathrm{p}<0.0001$, $\mathrm{t}$-test). There was no statistically significant difference between the ROS levels in the two diabetic groups ( $\mathrm{p} \geq 0.05)$.

Fig. 1. ROS levels expressed in arbitrary units in controls, patients with poor and good glycemic control

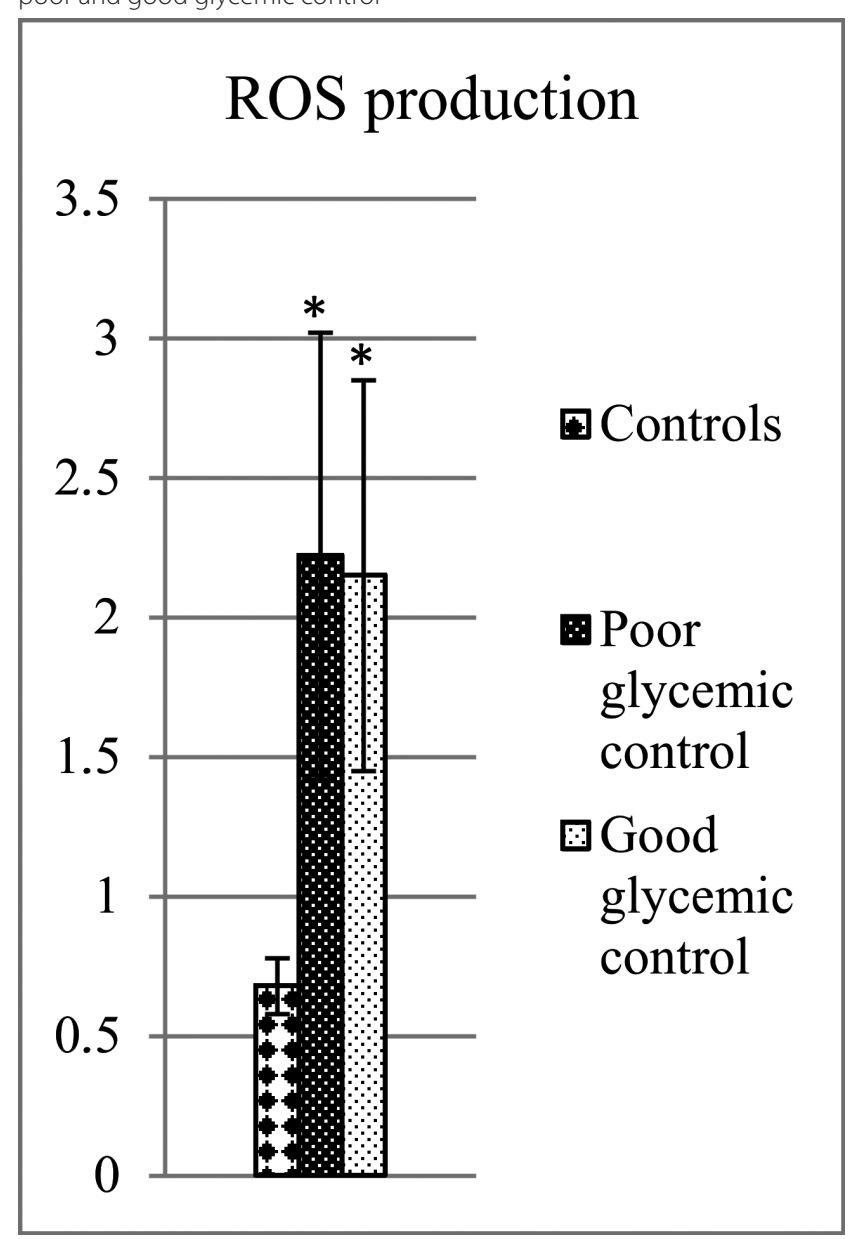

$p<0.0001 ;{ }^{*}$ - statistically significant compared to controls. 
Fig. 2 shows decreased levels of the Asc $\bullet$ radical in poorly controlled diabetic patients compared to the controls (mean $0.21 \pm 0.03$, vs mean $0.28 \pm 0.06$, p > 0.05, $\mathrm{t}$-test). A similar decrease was observed in the well-controlled group compared to the controls (mean $0.20 \pm 0.08$,

Fig. 2. Asc. radical levels measured in the serum of healthy controls and diabetic patients with poor and good glycemic control, expressed in arbitrary units

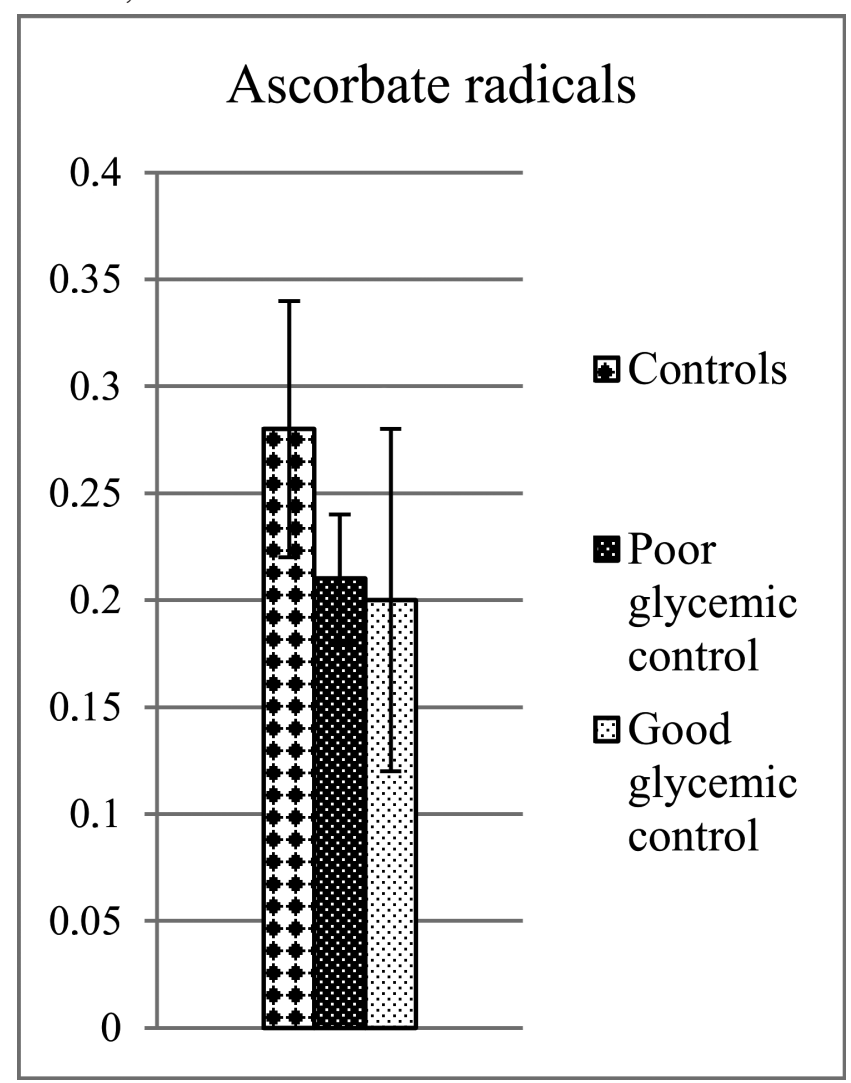

Fig. 3. Levels of $\cdot \mathrm{NO}$ expressed in arbitrary units in controls, the group with poor glycemic control and the group with good glycemic control

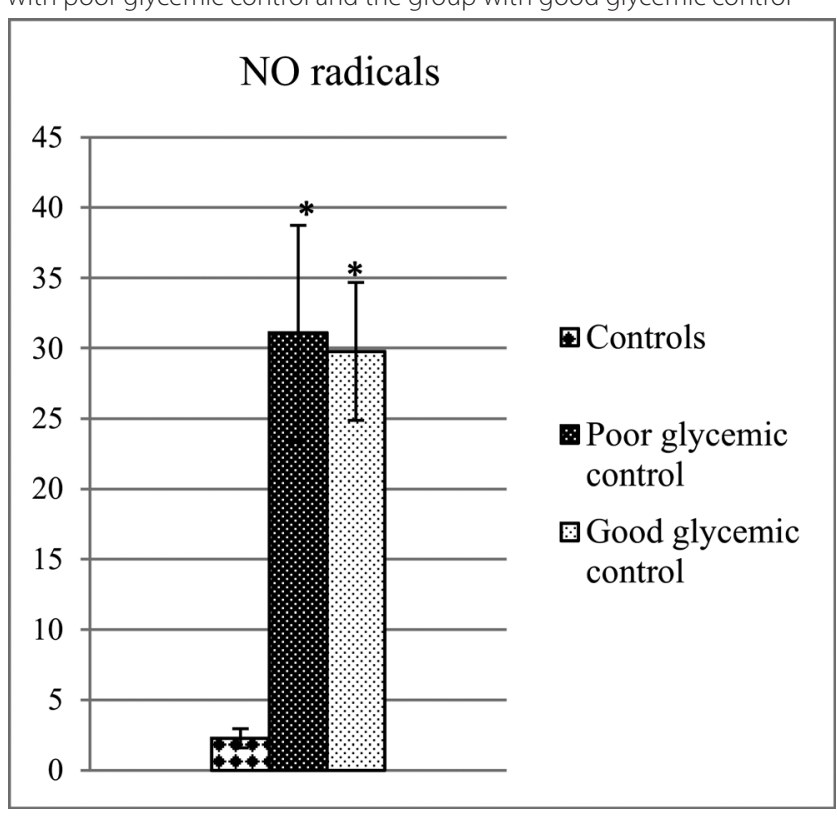

$p<0.0001 ;^{*}$ - statistically significant, increases compared to the controls. $\mathrm{p} \geq 0.05$, t-test). No statistically significant difference was observed in Asc levels between the 2 groups of diabetic patients $(\mathrm{p} \geq 0.05)$.

Fig. 3 shows statistically significant increases in the levels of registered $\cdot \mathrm{NO}$ radicals in the diabetic patients with poor glycemic control compared to the controls (mean $31.09 \pm 7.67$ vs mean $2.26 \pm 0.67$, p $<0.0001$, t-test). The same was observed in the well-controlled group compared to the controls (mean $29.78 \pm 4.90$, p $<0.0001$, t-test). There was no statistically significant difference between the levels of $\bullet \mathrm{NO}$ radicals in patients between the 2 groups ( $\mathrm{p} \geq 0.05)$.

\section{Correlations between clinical and biochemical parameters}

The serum •NO levels significantly correlated with average blood glucose levels $(\bullet \mathrm{NO}$ vs $\mathrm{HbA} 1, \mathrm{r}=0.54$, $\mathrm{p}=0.000)$, lipid levels $(\cdot \mathrm{NO}$ vs total cholesterol, $\mathrm{r}=0.33$, $\mathrm{p}=0.000 ; \cdot \mathrm{NO}$ vs HDL, $\mathrm{r}=0.37, \mathrm{p}=0.006 ; \cdot \mathrm{NO}$ vs LDL, $\mathrm{r}=0.32, \mathrm{p}=0.00)$, and triglycerides $(\mathrm{r}=0.35, \mathrm{p}=0.00)$.

The serum ROS levels show a positive correlation with average blood glucose levels $(\mathrm{r}=0.42, \mathrm{p}=0.000)$. Also, ROS levels significantly correlated with triglycerides (ROS vs triglycerides, $\mathrm{r}=0.20, \mathrm{p}=0.5$ ), total cholesterol and its fractions (ROS vs total cholesterol $r=0.23$, $\mathrm{p}=0.09$; ROS vs HDL, $\mathrm{r}=0.36, \mathrm{p}=0.005$; ROS vs LDL, $\mathrm{r}=0.20, \mathrm{p}=0.065)$.

\section{Discussion}

Regardless of the primary causes of type 2 diabetes, the common clinical course for patients is to respond initially to therapy by normalizing their fasting glucose levels, but then to undergo gradual deterioration in glycemic control, despite optimal medical management, using a variety of drugs. The initial improvement in fasting glucose may be misleading since many patients still have very elevated glucose levels postprandial. This suggests the idea of glucose toxicity, as the prolonged exposure to very high blood glucose levels can cause toxic effects on the $\beta$-cells and chronic hyperglycemia, and explains the abnormal lipid level. ${ }^{16}$ Chronic hyperglycemia may damage the tissue of diabetes patients by increasing the glucose flow, intracellular inclusions, and hyperactivity of the hexosamine pathway. All these processes are activated by mitochondrial ROS overproduction. ${ }^{17}$ The major consequences of hyperglycemia in diabetics are the microvascular and macrovascular complications. Microvascular pathogenesis is established with the chemical reaction between the by-products of sugars and proteins, while the macrovascular complications are multifactorial because diabetes at first impairs the ability of the vascular endothelium to vasodilate through $\bullet \mathrm{NO}$ initiation, which increases the oxidation of fatty acids and 
inhibits the production of enzymes responsible for clotting, and increases ROS generation, partially resulting from pathway specific insulin resistance. ${ }^{18}$

Hyperglycemia can be controlled clinically through insulin administration or through drugs which increase insulin secretion, decrease glucose release from the liver, increase the use of glucose in the skeletal muscle and fat, delay glucose absorption from foods, and most recently, act through the incretin system. These advances, together with improved glucose monitoring and better markers of glycemic control, have led to much tighter control of hyperglycemia. ${ }^{19}$ There is a large number of studies, which prove that early intensive glycemic control reduces the risk of diabetic complications. ${ }^{20,21}$ Hyperglycemia induces oxidative stress as well as ROS activation.

This study explored the influence of glycemic control on some real-time biomarkers of free radical formation in type 2 diabetic patients, using an EPR method. The $1^{\text {st }}$ marker for free radical formation in this study was the ROS levels estimated in both patient groups studied, i.e., with poor glycemic control and with good glycemic control compared to the healthy controls. In the course of lipid peroxidation, a variety of unstable ROS are formed, and can be measured using the EPR spin trapping technique. ${ }^{22,23}$ To establish the serum oxidative status from the controls and diabetic patients in both groups, we used a spin trap PBN agent.

Our results indicate statistically significantly higher ROS product levels ( $\mathrm{p}<0.0001)$ in type 2 diabetic patients in both groups compared to the controls, which means that in these patients oxidative processes take place at the time of the survey. It should be noted that after the adjustment of blood glucose levels by intensified insulin therapy (4 times/per day administration of insulin Actrapid HM) in hospital, the levels of ROS products in the group with well-controlled diabetes were slightly higher compared to the group with poorly controlled diabetes. Moreover, overproduction of the free radicals persists after the normalization of the glucose levels, and oxidative stress may be involved in the "metabolic memory" effect. ${ }^{24}$ The "metabolic memory" phenomenon was first observed in preclinical studies, and was later confirmed in clinical trials - it describes the beneficial effects of immediate treatment of hyperglycemia. Along with OS and chronic hyperglycemia, the mitochondria are an important player in propagating "metabolic memory". Potential mechanisms for propagating this "memory" are the non-enzymatic glycation of cellular proteins and lipids and an excess of cellular ROS/RNS, in particular originating at the level of glycated-mitochondrial proteins, perhaps acting in concert with one another to maintain stress signaling. The formation of advanced glycation end-products (AGEs) in the mitochondria plays a crucial role in diabetic complications. Thus, OS may be involved in sup- porting "metabolic memory", and describes the relationship between the development of complications of diabetes and prolonged exposure to glucotoxicity. The cycle of events in the vascular cells might suggest that OS, associated with delayed therapeutic intervention, is essential in "metabolic memory". Hyperglycemic memory can also appear even when good glycemic control is achieved, and this is evidenced by the persistent OS markers after reaching normal levels of blood glucose. In endothelial cells, the overproduction of free radicals persists after glucose normalization. Apart from this, the epidemiological and prospective data support a long-term influence of early intensive metabolic control on clinical outcomes and reduce the risk of diabetic complications. ${ }^{19}$ Moreover, the emergence of this "metabolic memory" suggests the need of very early aggressive treatment aiming to normalize metabolic control and the addition of agents which reduce cellular ROS and glycation in order to minimize longterm diabetic complications. When high blood glucose is corrected, the adverse effects are not reversed, and some may be permanent because of epigenetic changes. In addition, biomarkers that link the short-term, realtime and "metabolic memory" markers resulting from long-term hyperglycemia and hiperlipidemia-induced OS can be valuable for predicting not only vascular complications in type 2 diabetes, but also the onset of diabetes. This conforms with the positive correlation between ROS levels and average blood glucose levels, triglycerides, and total cholesterol with its fractions (HDL, LDL).

A number of physiological active molecules can be modified by ROS to stable organic radicals. ${ }^{25}$ In biological conditions, ROS reacts with ascorbic acid and produces the Asc radical. The relatively long lifespan of the Asc radical makes it a natural indicator for assessing the oxidative status in living organisms. ${ }^{26-29}$ The low levels of the Asc radical measured in the serum in both diabetic groups may be the result of a partial depletion of available vitamin $C$ reserves compared to the healthy controls. This can be due to the accumulation of free radicals, which contributes to an increase in oxidation processes in the subjects studied. It is well-known that water soluble ascorbic acid acts as a chain breaking antioxidant and in biological systems scavenges free radicals such as ROS by donating electrons, and thus may prevent other biological molecules from being oxidized..$^{30,31}$

It is obvious that ROS and RNS $\left(\mathrm{ONOO}^{-}\right)$can damage a cell by biochemical oxidation of lipids, proteins, and DNA. Nitric oxide $(\bullet N O)$ is a unique biological messenger molecule which plays diverse physiologic roles. •NO mediates blood vessel relaxation, the immune activity of macrophages and the neurotransmission of central and peripheral neurons. ${ }^{32}$ It is believed that free radicals are directly implicated in the pathogenesis of diabetes and that levels of $\bullet \mathrm{NO}$ radicals in type 2 diabetes patients are increased. ${ }^{33-35}$ 
A number of studies have demonstrated lower levels of nitrite/nitrate plasma in diabetic patients compared to the healthy controls. ${ }^{36,37}$ Diabetic patients often experience elevated glucose levels. A part of this glucose becomes incorporated into hemoglobin and is measured as glycosylated hemoglobin (HbA1c). Glycosylated hemoglobin binds -NO very tightly in the form of nitrosothiols and any $\bullet \mathrm{NO}$ that is formed cannot be easily released from red blood cells. ${ }^{36,37}$ Limited release of $\bullet \mathrm{NO}$ is one of the reasons for violating essential cellular functions. Since in our study (Fig. 3) Carboxy-PTIO has been used as a spin trapping agent for the $\bullet \mathrm{NO}$ radical, we consider that the value of $\bullet \mathrm{NO}$ measured in the serum of diabetic patients is not only that measured as nitrite/nitrate, but also •NO linked with glycated hemoglobin. These results were supported by positive correlations with clinical parameters (HbAlc, blood glucose, triglycerides, total cholesterol, HDL- and LDL-cholesterol). On the other hand, the increased levels of $\bullet \mathrm{NO}$ and ROS reported in this study probably lead to the generation of toxic $\mathrm{ONOO}^{-}$, which may play a significant role in the pathogenesis of diabetes and its complications.

We can speculate that hyperglycemia leads to increased production of free radicals, increased oxidative stress, and disturbances in antioxidant protection, which might be a factor for the initiation and development of complications in diabetes mellitus. ${ }^{38}$ Moreover, higher levels of real-time biomarkers show that intensive insulin treatment does not lead to the expected decrease in oxidative processes involving ROS and $\bullet \mathrm{NO}$, probably due to "metabolic memory". Given the key role of the cascade of oxidative stress and "metabolic memory", very early aggressive treatment aiming to normalize glycemic control is needed to reduce cellular reactive species and glycation, and to minimize long-term diabetic complications.

\section{References}

1. Halliwell B, Whiteman M. Measuring reactive species and oxidative damage in vivo and in cell culture: How should you do it and what do the results mean? Br J Pharmacol. 2004;142:231-255.

2. Gadjeva V. Oxidative stress, cancer and chemotherapy. Ac Publ Tr Univer St. Zagora. 2007.

3. Shin CS, Moon BS, Park KS, et al. Serum 8-hydroxy-guanine levels are increased in diabetic patients. Diabetes. 2001;24:733-737.

4. Sakuraba H, Mizukami H, Yagihashi N, Wada R, Hanyu C, Yagihashi S. Reduced $\beta$-cell mass and expression of oxidative stress related DNA damage in the islet of Japanese type II diabetic patients. Diabetologia. 2002;45:85-96.

5. Halliwell B, Gutteridge JMC. Free radicals in biology and medicine. $5^{\text {th }}$ ed. New York, NY: Oxford University Press; 2015.

6. Nowotny K, Jung T, Höhn A, Weber D, Grune T. Advanced glycation end products and oxidative stress in type 2 diabetes mellitus. Biomolecules. 2015;5:194-222.

7. Pitocco D, Tesauro M, Alessandro R, Ghirlanda G, Cardillo C. Oxidative stress in diabetes: Implications for vascular and other complications. IJMS. 2013;14:21525-21550.

8. Gruden G, Barutta F, Kunos G, Pacher P. Role of the endocannabinoid system in diabetes and diabetic complications. Br J Pharmacol. 2016;173(7):1116-1127.

9. Ido Y. Diabetic complications within the context of ageing: $\mathrm{NADH} / \mathrm{NAD}+$ redox, insulin C-peptide, SIRT1-LKB1-AMPK positive feedback and FOXO3. J Diabetes Investig. 2016;7(4):448-458.
10. Giacco F, Brownlee M. Oxidative stress and diabetic complications. Circulation research. 2010,107:1058-1070.

11. Zheleva A. Electron paramagnetic resonance-oxidative status and antioxidant activity. Ac Publ Tr Univer St. Zagora. 2012:44-55.

12. Bailey DM. Ascorbate, blood-brain barrier function and acute mountain sickness: A radical hypothesis. Wilderness \& environmental medicine. 2004;15:231-233.

13. Shi $H$, Sui $Y$, Wang $X$, Luo Yi, Ji L. Hydroxyl radical production and oxidative damage induced by cadmium and naphthalene in liver of Carassius auratus. Comp Biochem Physiol Part C Toxicol Pharmcol. 2005;140:115-121.

14. Yoshioka T, Iwamoto N, Ito K. An application of electron paramagnetic resonance to evaluate nitric oxide and its quenchers. J Am Soc Nephrol. 1996;7:961-965.

15. Yokoyama K, Hashiba K, Wakabayashi H, et al. Inhibition of LPSstimulated NO production in mouse macrophage-like cells by Tropolones.Anticancer Research. 2004;24:3917-3922.

16. Robertson RP, Harmon J, Tran PO, Poitout V. $\beta$-cell glucose toxicity, lipotoxicity, and chronic oxidative stress in type 2 diabetes. Diabetes. 2004;53:119-124.

17. Brownlee M. The pathobiology of diabetic complications: A unifying mechanism. Diabetes. 2005;54:1615-1625.

18. Cade WT. Diabetes-related microvascular and macrovascular diseases in the physical therapy setting. Physical Therapy. 2008;88:1322-1335.

19. Ceriello A. The hyperglycemia-induced metabolic memory: The new challenge for the prevention of CVD in diabetes. Revista Española de Cardiología. 2008;8(Suppl C):11-17.

20. Brownlee M. The pathobiology of diabetic complications: A unifying mechanism. Diabetes. 2005;54(6):1615-1625.

21. Piwowar A, Knapik-Kordecka M, Warwas M. AOPP and its relations with selected markers of oxidative/antioxidative system in type 2 diabetes mellitus. Diabetes Res Clin Pract. 2007,77(2):188-192.

22. Mason RP, Hanna PM, Burkitt MJ, Kadiiska MB. Detection of oxygen-derived radicals in biological systems using electron spin resonance. Environ Health Perspect. 1994;102:33.

23. Luo Y, Roth GS. The roles of dopamine oxidative stress and dopamine receptor signaling in aging and age-related neurodegeneration. ARS. 2000;2:449-460.

24. Aschner PJ, Ruiz AJ. Metabolic memory for vascular disease in diabetes. Diabetes Technol Ther. 2012;14(Suppl 1):68-74.

25. Takeshita K, Fujii K, Anzai K, Ozawa T. In vivo monitoring of hydro$x y l$ radical generation caused by $X$-ray irradiation of rats using the spin trapping/EPR technique. Free Rad Biol Med. 2004;36:1134-1143.

26. Armstrong D. Oxidative Stress Biomarkers and Antioxidant Protocols. Totowa, NJ: Humana Press; 2002:186.

27. Buettner GR, Jurkiewicz BA. Ascorbate free radical as a marker of oxidative stress: An EPR study. Free Rad Biol and Med. 1993;14:49-55.

28. Nakagawa K, Kanno H, Miura Y. Detection and analyses of ascorbyl radical in cerebrospinal fluid and serum of acute lymphoblastic leukemia. Anal Biochem. 1997;254:31-35.

29. Chen Q, Espey MG, Sun AY, et al. Ascorbate in pharmacologic concentrations selectively generates ascorbate radical and hydrogen peroxide in extracellular fluid in vivo. Proc Natl Acad Sci USA. 2007;104:8749-8754.

30. Buettner GR. Workshop: Rigorous Detection and Identification of Free Radicals in Biology and Medicine. SFRBM 2015, November. www.sfrbm.org/frs/Buettner2005.ppt.

31. Asmat U, Abad K, Ismail K. Diabetes mellitus and oxidative stress: A concise review. Saudi Pharm J. 2016;24(5):547-553.

32. Vinish $M$, Anand A, Prabhakar S. Altered oxidative stress levels in Indian Parkinson's disease patients with PARK2 mutations. Acta Biochim Pol. 2011;58:165-169.

33. Giacco F, Brownlee M. Oxidative stress and diabetic complications. Circ Res. 2010;107:1058-1070.

34. Chang Yi-Ch, Chuang Lee-M. The role of oxidative stress in the pathogenesis of type 2 diabetes: From molecular mechanism to clinical implication. Am J Transl Res. 2010;2:316-331.

35. Henry Y, Guissani A. Contribution of spin-trapping EPR techniques for the measurement of NO production in biological systems. Analusis. 2000;28:445-454

36. Gradinaru D, Borsa C, lonescu C, Margina D. Advanced oxidative and glycoxidative protein damage markers in the elderly with type 2 diabetes. Journal of Proteomics. 2013;92:313-322. 
37. Gradinaru D, Borsa C, lonescu C, Prada GI. Oxidized LDL and NO synthesis - biomarkers of endothelial dysfunction and ageing. Mech Ageing Dev. 2015;151:101-113.

38. Monnier L, Colette C, Michel F, Cristol JP, Owens DR. Insulin therapy has a complex relationship with measure of oxidative stress in type 2 diabetes: A case for further study. Diab Metab Res Rev. 2011;27:348-353. 